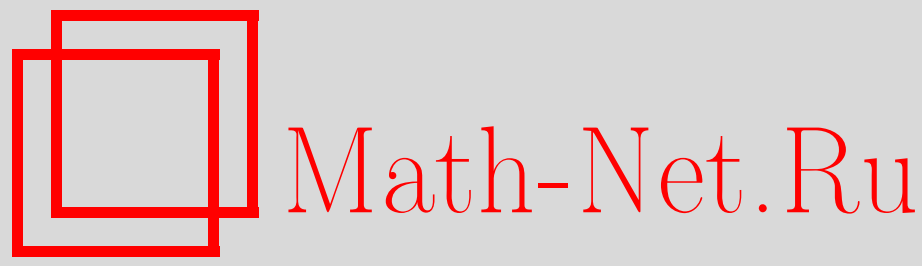

О. И. Мохов, О согласованных метриках и диагонализуемости нелокально-бигамильтоновых систем гидродинамического типа, ТМФ, 2011, том 167, номер 1, 3-22

DOI: https://doi.org/10.4213/tmf6623

Использование Общероссийского математического портала Math-Net.Ru подразумевает, что вы прочитали и согласны с пользовательским соглашением http://www.mathnet.ru/rus/agreement

Параметры загрузки:

IP : 54.164 .48 .24

26 апреля 2023 г., 08:09:10

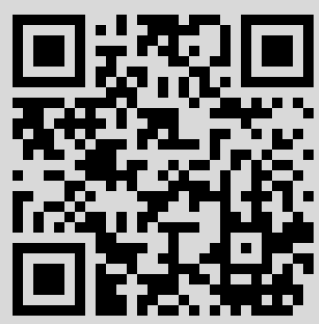




\title{
ФИЗИКА
}

Том 167, № 1

апрель, 2011

(C) 2011 г. $\quad$ О. И. Мохов*† $^{* \dagger}$

\section{О СОГЛАСОВАННЫХ МЕТРИКАХ И ДИАГОНАЛИЗУЕМОСТИ НЕЛОКАЛЬНО-БИГАМИЛЬТОНОВЫХ СИСТЕМ ГИДРОДИНАМИЧЕСКОГО ТИПА}

\begin{abstract}
Изучаются бигамильтоновы системы гидродинамического типа с неособыми (полупростыми) нелокальными бигамильтоновыми структурами. Доказано, что все такие системы гидродинамического типа являются диагонализуемыми, причем построенный для произвольной такой системы полный набор римановых инвариантов полностью определяется метриками бигамильтоновой структуры. Более того, доказано, что для произвольной неособой (полупростой) нелокально-бигамильтоновой системы гидродинамического типа существуют локальные координаты (римановы инварианты) такие, что все связанные с данной системой матричные дифференциально-геометрические объекты, а именно матрица (аффинор) $V_{j}^{i}(u)$ этой системы гидродинамического типа, метрики $g_{1}^{i j}(u)$ и $g_{2}^{i j}(u)$, аффинор $v_{j}^{i}(u)=g_{1}^{i s}(u) g_{2, s j}(u)$, а также аффиноры $\left(w_{1, n}\right)_{j}^{i}(u)$ и $\left(w_{2, n}\right)_{j}^{i}(u)$ неособой нелокальной бигамильтоновой структуры этой системы, являются диагональными в этих специальных "диагонализующих" локальных координатах (римановых инвариантах системы). Доказательство является естественным следствием общих результатов теории согласованных метрик и теории нелокальных бигамильтоновых структур, развитых ранее автором; приводится краткий обзор необходимых понятий и результатов этих теорий.
\end{abstract}

Ключевые слова: бигамильтонова система гидродинамического типа, римановы инварианты, согласованные метрики, диагонализуемый аффинор, бигамильтонова структура, бигамильтонов аффинор, интегрируемая система.

\section{1. ВВЕДЕНИЕ}

Мы рассматриваем $(1+1)$-мерные неособые (полупростые) нелокально-бигамильтоновы системы гидродинамического типа и доказываем их диагонализуемость. Для произвольной такой системы построен полный набор римановых инвариантов, который полностью определяется метриками бигамильтоновой структуры. Более того,

${ }^{*}$ Институт теоретической физики им. Л. Д. Ландау РАН, Черноголовка, Московская обл., Россия. E-mail: mokhov@landau.ac.ru

${ }^{\dagger}$ Московский государственный университет им. М. В. Ломоносова, Москва, Россия. E-mail:mokhov@mi.ras.ru,mokhov@bk.ru 
доказано, что для произвольной неособой (полупростой) нелокально-бигамильтоновой системы гидродинамического типа существуют локальные координаты (римановы инварианты) такие, что все связанные с данной системой матричные дифференциально-геометрические объекты, а именно матрица (аффинор) $V_{j}^{i}(u)$ этой системы гидродинамического типа, метрики $g_{1}^{i j}(u)$ и $g_{2}^{i j}(u)$, аффинор $v_{j}^{i}(u)=g_{1}^{i s}(u) g_{2, s j}(u)$, а также аффиноры $\left(w_{1, n}\right)_{j}^{i}(u)$ и $\left(w_{2, n}\right)_{j}^{i}(u)$ неособой нелокальной бигамильтоновой структуры этой системы, являются диагональными в этих специальных "диагонализующих" локальных координатах (римановых инвариантах системы). Отметим, что все утверждения, конечно же, остаются верными и в частном случае, когда одна или обе согласованные гамильтоновы структуры бигамильтоновой системы являются локальными, но при этом доказательства упрощаются. Для локальных неособых бигамильтоновых структур диагонализуемость системы является прямым следствием теории неособых (полупростых) согласованных плоских метрик. Нетривиальным является обобщение на нелокальный случай, для чего нам пришлось развить соответствующие теории нелокальных бигамильтоновых структур гидродинамического типа и согласованных общих псевдоримановых метрик. Мы приводим здесь очень кратко необходимые основные понятия и результаты. Напомним, что $(1+1)$-мерные системы гидродинамического типа [1] - это произвольные $(1+1)$-мерные эволюционные квазилинейные системы дифференциальных уравнений в частных производных первого порядка, т. е. уравнения вида

$$
u_{t}^{i}=V_{j}^{i}(u) u_{x}^{j}, \quad 1 \leqslant i, j \leqslant N,
$$

где $u=\left(u^{1}, \ldots, u^{N}\right)$ - локальные координаты на некотором гладком $N$-мерном многообразии или в области $\mathbb{R}^{N}$ (или $\left.\mathbb{C}^{N}\right) ; u^{i}(x)$ - функции (поля) одной переменной $x$, которые эволюционируют по $t ; V_{j}^{i}(u)$ - произвольная $(N \times N)$-матрица, зависящая от $u$ (эта матрица является смешанным тензором типа $(1,1)$, т. е. аффинором, относительно локальных замен координат $u)$. Функция $R\left(u^{1}, \ldots, u^{N}\right)$ называется $p u м a-$ новым инвариантом системы гидродинамического типа (1), если существует функция $\alpha\left(u^{1}, \ldots, u^{N}\right)$ такая, что в силу системы (1) мы имеем

$$
R_{t}=\alpha(u) R_{x} .
$$

С любым римановым инвариантом $R\left(u^{1}, \ldots, u^{N}\right)$ системы гидродинамического типа связана естественная редукция системы вида $R\left(u^{1}, \ldots, u^{N}\right)=$ const. Система гидродинамического типа является диагонализуемой тогда и только тогда, когда эта система обладает полным набором римановых инвариантов, т. е. множеством $N$ различных и функционально независимых римановых инвариантов, которые могут быть взяты в качестве новых локальных координат $\left\{R^{1}, \ldots, R^{N}\right\}, R^{i}=$ $R^{i}\left(u^{1}, \ldots, u^{N}\right), i=1, \ldots, N$. В римановых инвариантах система становится диагональной:

$$
R_{t}^{i}=\alpha^{i}(R) R_{x}^{i}, \quad i=1, \ldots, N .
$$

\section{2. НЕЛОКАЛЬНО-ГАМИЛЬТОНОВЫ СИСТЕМЫ ГИДРОДИНАМИЧЕСКОГО ТИПА}

Мы будем рассматривать системы вида (1), которые являются гамильтоновыми относительно произвольных невырожденных нелокальных скобок Пуассона гидродинамического типа (скобок Ферапонтова [2]; см. также [3] и [1] для скобок Мохова-Ферапонтова и скобок Дубровина-Новикова в специальном нелокальном и чисто 
локальном случаях соответственно), т. е.

$$
u_{t}^{i}=V_{j}^{i}(u) u_{x}^{j}=\left\{u^{i}(x), H\right\}, \quad 1 \leqslant i, j \leqslant N,
$$

где функционал

$$
H=\int h(u(x)) d x
$$

является гамильтонианом системы (4) (функция $h(u)$ - плотность гамильтониана), а скобка Пуассона имеет вид

$$
\begin{gathered}
\left\{u^{i}(x), u^{j}(y)\right\}=P^{i j} \delta(x-y), \quad 1 \leqslant i, j \leqslant N, \\
P^{i j}=g^{i j}(u(x)) \frac{d}{d x}+b_{k}^{i j}(u(x)) u_{x}^{k}+ \\
+\sum_{m, n=1}^{L} \mu^{m n}\left(w_{m}\right)_{k}^{i}(u(x)) u_{x}^{k}\left(\frac{d}{d x}\right)^{-1} \circ\left(w_{n}\right)_{s}^{j}(u(x)) u_{x}^{s},
\end{gathered}
$$

где коэффициенты $g^{i j}(u), b_{k}^{i j}(u)$ и $\left(w_{n}\right)_{j}^{i}(u), 1 \leqslant i, j, k \leqslant N, 1 \leqslant n \leqslant L,-$ гладкие функции локальных координат, $\operatorname{det}\left(g^{i j}(u)\right) \neq 0, \mu^{m n}-$ произвольная невырожденная симметричная постоянная матрица, $\mu^{m n}=\mu^{n m}, \mu^{m n}=\mathrm{const}, \operatorname{det}\left(\mu^{m n}\right) \neq 0$. Для двух произвольных функционалов $I$ и $J$ скобка Пуассона $(6),(7)$ имеет вид

$$
\{I, J\}=\int \frac{\delta I}{\delta u^{i}(x)} P^{i j} \frac{\delta J}{\delta u^{j}(x)} d x .
$$

Скобки Пуассона вида (7), (8) были введены и изучены Ферапонтовым в работе [2]; эти скобки являются нелокальным обобщением скобок Дубровина-Новикова [1] (локальных скобок Пуассона гидродинамического типа, порождаемых плоскими метриками $g^{i j}(u)$; в этом случае нет нелокальных членов, $L=0$, или $\left.\left(w_{n}\right)_{j}^{i}(u)=0\right)$ и скобок Мохова-Ферапонтова [3] (нелокальных скобок Пуассона гидродинамического типа, порождаемых метриками постоянной кривизны $K$; в этом случае $L=1$, $\left.\mu^{11}=K,\left(w_{1}\right)_{j}^{i}(u)=\delta_{j}^{i}\right)$. Ферапонтов доказал, что нелокальный оператор $P^{i j}$ вида (7) задает скобку Пуассона (8) тогда и только тогда, когда существует $N$-мерное подмногообразие с плоской нормальной связностью в $(N+L)$-мерном псевдоевклидовом пространстве такое, что $g^{i j}(u)$ - контравариантная первая квадратичная форма; $b_{k}^{i j}(u)=-g^{i s}(u) \Gamma_{s k}^{j}(u) ; \Gamma_{s k}^{j}(u)$ - символы Кристоффеля связности Леви-Чивиты метрики $g^{i j}(u) ;\left(w_{n}\right)_{j}^{i}(u), 1 \leqslant n \leqslant L,-$ операторы Вейнгартена (аффиноры Вейнгартена) подмногообразия; $\mu^{m n}$ - матрица Грама соответствующих параллельных базисов в нормальных пространствах подмногообразия (все формы кручения подмногообразия с плоской нормальной связностью тождественно равны нулю в этих базисах в нормальных пространствах).

Другими словами, нелокальный оператор (7) является гамильтоновым, т. е. задает скобку Пуассона (8), тогда и только тогда, когда его коэффициенты удовлетворяют соотношениям (см. также [4])

$$
\begin{aligned}
g^{i j} & =g^{j i}, \\
\frac{\partial g^{i j}}{\partial u^{k}} & =b_{k}^{i j}+b_{k}^{j i},
\end{aligned}
$$




$$
\begin{aligned}
& g^{i s} b_{s}^{j k}=g^{j s} b_{s}^{i k}, \\
& g^{i s}\left(w_{n}\right)_{s}^{j}=g^{j s}\left(w_{n}\right)_{s}^{i}, \\
& \left(w_{n}\right)_{s}^{i}\left(w_{m}\right)_{j}^{s}=\left(w_{m}\right)_{s}^{i}\left(w_{n}\right)_{j}^{s} \text {, } \\
& g^{i s} g^{j r} \frac{\partial\left(w_{n}\right)_{r}^{k}}{\partial u^{s}}-g^{j r} b_{s}^{i k}\left(w_{n}\right)_{r}^{s}=g^{j s} g^{i r} \frac{\partial\left(w_{n}\right)_{r}^{k}}{\partial u^{s}}-g^{i r} b_{s}^{j k}\left(w_{n}\right)_{r}^{s} \text {, } \\
& g^{i s}\left(\frac{\partial b_{s}^{j k}}{\partial u^{r}}-\frac{\partial b_{r}^{j k}}{\partial u^{s}}\right)+b_{s}^{i j} b_{r}^{s k}-b_{s}^{i k} b_{r}^{s j}= \\
& =\sum_{m=1}^{L} \sum_{n=1}^{L} \mu^{m n} g^{i s}\left(\left(w_{m}\right)_{r}^{j}\left(w_{n}\right)_{s}^{k}-\left(w_{m}\right)_{s}^{j}\left(w_{n}\right)_{r}^{k}\right) \text {. }
\end{aligned}
$$

\section{3. НЕЛОКАЛЬНО-ГАМИЛЬТОНОВЫ АФФИНОРЫ}

Гамильтониан $H$ системы (4)-(8) должен также быть первым интегралом всех систем гидродинамического типа, которые задаются аффинорами $\left(w_{n}\right)_{j}^{i}(u), 1 \leqslant n \leqslant$ $L$, нелокального оператора (7) (эти системы называются структурными потоками нелокальной скобки Пуассона (6)-(8)) [2]:

$$
u_{t_{n}}^{i}=\left(w_{n}\right)_{j}^{i}(u) u_{x}^{j}, \quad H_{t_{n}}=0, \quad 1 \leqslant n \leqslant L .
$$

Для каждого $n, 1 \leqslant n \leqslant L$, существует функция $f_{n}(u)$ такая, что

$$
\frac{\partial h}{\partial u^{j}}\left(w_{n}\right)_{s}^{j}(u)=\frac{\partial f_{n}}{\partial u^{s}}, \quad 1 \leqslant n \leqslant L .
$$

В этом случае аффинор $V_{j}^{i}(u)$ системы гидродинамического типа (4)-(8) имеет вид

$$
V_{j}^{i}(u)=g^{i s}(u) \frac{\partial^{2} h}{\partial u^{s} \partial u^{j}}-g^{i s}(u) \Gamma_{s j}^{p}(u) \frac{\partial h}{\partial u^{p}}+\sum_{m, n=1}^{L} \mu^{m n}\left(w_{m}\right)_{j}^{i}(u) f_{n}(u),
$$

T. e.

$$
\begin{aligned}
V_{j}^{i}(u) & =g^{i s}(u) \nabla_{s} \nabla_{j} h(u)+\sum_{m, n=1}^{L} \mu^{m n}\left(w_{m}\right)_{j}^{i}(u) f_{n}(u)= \\
& =\nabla^{i} \nabla_{j} h(u)+\sum_{m, n=1}^{L} \mu^{m n}\left(w_{m}\right)_{j}^{i}(u) f_{n}(u),
\end{aligned}
$$

где $\nabla_{k}$ - ковариантная производная, порождаемая связностью Леви-Чивиты $\Gamma_{s k}^{j}(u)$ метрики $g^{i j}(u)$.

Мы будем называть аффинор $V_{j}^{i}(u)$ гамильтоновым (или нелокально-гамильтоновым), если существуют $N$-мерное подмногообразие с плоской нормальной связностью в $(N+L)$-мерном псевдоевклидовом пространстве и функции $h(u)$ и $f_{n}(u)$, $1 \leqslant n \leqslant L$, такие, что аффинор $V_{j}^{i}(u)$ имеет вид $(19)$, где $g^{i j}(u)$ - контравариантная первая квадратичная форма подмногообразия; $\nabla_{k}$ - ковариантная производная, порождаемая связностью Леви-Чивиты $\Gamma_{s k}^{j}(u)$ метрики $g^{i j}(u) ;\left(w_{n}\right)_{j}^{i}(u), 1 \leqslant n \leqslant L,-$ 
операторы Вейнгартена подмногообразия; $\mu^{m n}$ - матрица Грама соответствующих параллельных базисов в нормальных пространствах подмногообразия (так что все формы кручения подмногообразия с плоской нормальной связностью тождественно равны нулю в этих базисах в нормальных пространствах), и функции $h(u)$ и $f_{n}(u)$, $1 \leqslant n \leqslant L$, удовлетворяют соотношениям (17):

$$
\left(w_{n}\right)_{s}^{j}(u) \nabla_{j} h(u)=\nabla_{s} f_{n}(u), \quad 1 \leqslant n \leqslant L .
$$

В этом случае мы будем также говорить, что аффинор $V_{j}^{i}(u)(19),(20)$ является гамильтоновым относительно соответствующей нелокальной скобки Пуассона гидродинамического типа (6)-(8). Очевидно, что это определение инвариантно. Отметим, что его можно также рассматривать в качестве определения нелокально-гамильтоновой системы гидродинамического типа (1).

Аффиноры, которые являются гамильтоновыми относительно скобок Дубровина-Новикова (локально-гамильтоновы аффиноры), были детально изучены Царевым в его замечательной работе [5]. Аффиноры, которые являются гамильтоновыми относительно нелокальных скобок Мохова-Ферапонтова (аффиноры Мохова-Ферапонтова), были детально изучены в работе [3].

ПреДЛожениЕ. Для нелокально-гамильтоновых аффиноров $V_{j}^{i}(u)$ всегда выполняются следующие соотношения:

$$
\begin{aligned}
g_{i s}(u) V_{j}^{s}(u) & =g_{j s}(u) V_{i}^{s}(u), \\
\nabla_{j} V_{k}^{i}(u) & =\nabla_{k} V_{j}^{i}(u),
\end{aligned}
$$

где $g_{i j}(u)$ - матрица, обратная матрице $g^{i j}(u), g_{i s}(u) g^{s j}(u)=\delta_{i}^{j}$ (ковариантная метрика).

Действительно, выражение $g_{i s}(u) V_{j}^{s}(u)$ симметрично по индексам $i$ и $j$ :

$$
\begin{gathered}
g_{i s}(u) V_{j}^{s}(u)=g_{i s}(u)\left(g^{s r}(u) \frac{\partial^{2} h}{\partial u^{r} \partial u^{j}}-g^{s r}(u) \Gamma_{r j}^{p}(u) \frac{\partial h}{\partial u^{p}}+\sum_{m, n=1}^{L} \mu^{m n}\left(w_{m}\right)_{j}^{s}(u) f_{n}(u)\right)= \\
=\frac{\partial^{2} h}{\partial u^{i} \partial u^{j}}-\Gamma_{i j}^{p}(u) \frac{\partial h}{\partial u^{p}}+\sum_{m, n=1}^{L} \mu^{m n} g_{i s}(u)\left(w_{m}\right)_{j}^{s}(u) f_{n}(u)=g_{j s}(u) V_{i}^{s}(u),
\end{gathered}
$$

где использовано соотношение (12), эквивалентное условию

$$
g_{i s}\left(w_{n}\right)_{j}^{s}=g_{j s}\left(w_{n}\right)_{i}^{s} .
$$

Докажем соотношение (22), т. е. симметричность по индексам $j$ и $k$ выражения $\nabla_{j} V_{k}^{i}(u)$. Имеем

$$
\begin{aligned}
\nabla_{j} V_{k}^{i}(u)= & \frac{\partial V_{k}^{i}}{\partial u^{j}}+\Gamma_{r j}^{i}(u) V_{k}^{r}(u)-\Gamma_{k j}^{r}(u) V_{r}^{i}(u)= \\
= & \frac{\partial g^{i s}}{\partial u^{j}} \frac{\partial^{2} h}{\partial u^{s} \partial u^{k}}+g^{i s}(u) \frac{\partial^{3} h}{\partial u^{s} \partial u^{k} \partial u^{j}}-\frac{\partial g^{i s}}{\partial u^{j}} \Gamma_{s k}^{p}(u) \frac{\partial h}{\partial u^{p}}- \\
& -g^{i s}(u) \frac{\partial \Gamma_{s k}^{p}}{\partial u^{j}} \frac{\partial h}{\partial u^{p}}-g^{i s}(u) \Gamma_{s k}^{p}(u) \frac{\partial^{2} h}{\partial u^{p} \partial u^{j}}+
\end{aligned}
$$




$$
\begin{aligned}
& +\sum_{m, n=1}^{L} \mu^{m n} \frac{\partial\left(w_{m}\right)_{k}^{i}}{\partial u^{j}} f_{n}(u)+\sum_{m, n=1}^{L} \mu^{m n}\left(w_{m}\right)_{k}^{i}(u) \frac{\partial f_{n}}{\partial u^{j}}+ \\
& +\Gamma_{r j}^{i}(u) g^{r s}(u) \frac{\partial^{2} h}{\partial u^{s} \partial u^{k}}-\Gamma_{r j}^{i}(u) g^{r s}(u) \Gamma_{s k}^{p}(u) \frac{\partial h}{\partial u^{p}}+ \\
& +\Gamma_{r j}^{i}(u) \sum_{m, n=1}^{L} \mu^{m n}\left(w_{m}\right)_{k}^{r}(u) f_{n}(u)-\Gamma_{k j}^{r}(u) g^{i s}(u) \frac{\partial^{2} h}{\partial u^{s} \partial u^{r}}+ \\
& +\Gamma_{k j}^{r}(u) g^{i s}(u) \Gamma_{s r}^{p}(u) \frac{\partial h}{\partial u^{p}}-\Gamma_{k j}^{r}(u) \sum_{m, n=1}^{L} \mu^{m n}\left(w_{m}\right)_{r}^{i}(u) f_{n}(u) .
\end{aligned}
$$

Некоторые слагаемые в выражении (25) очевидным образом симметричны по индексам $j$ и $k$, например в силу симметричности связности Леви-Чивиты: $\Gamma_{k j}^{r}(u)=$ $\Gamma_{j k}^{r}(u)$. Докажем симметричность по индексам $j$ и $k$ остальных частей выражения (25). Используя соотношение $(10)$, где $b_{k}^{i j}(u)=-g^{i s}(u) \Gamma_{s k}^{j}(u)$, получаем

$$
\begin{aligned}
\frac{\partial g^{i s}}{\partial u^{j}} & \frac{\partial^{2} h}{\partial u^{s} \partial u^{k}}-g^{i s}(u) \Gamma_{s k}^{p}(u) \frac{\partial^{2} h}{\partial u^{p} \partial u^{j}}+\Gamma_{r j}^{i}(u) g^{r s}(u) \frac{\partial^{2} h}{\partial u^{s} \partial u^{k}}= \\
= & \left(-g^{i p}(u) \Gamma_{p j}^{s}(u)-g^{s p}(u) \Gamma_{p j}^{i}(u)\right) \frac{\partial^{2} h}{\partial u^{s} \partial u^{k}}- \\
& -g^{i s}(u) \Gamma_{s k}^{p}(u) \frac{\partial^{2} h}{\partial u^{p} \partial u^{j}}+\Gamma_{r j}^{i}(u) g^{r s}(u) \frac{\partial^{2} h}{\partial u^{s} \partial u^{k}}= \\
= & -g^{i p}(u) \Gamma_{p j}^{s}(u) \frac{\partial^{2} h}{\partial u^{s} \partial u^{k}}-g^{i s}(u) \Gamma_{s k}^{p}(u) \frac{\partial^{2} h}{\partial u^{p} \partial u^{j}},
\end{aligned}
$$

что очевидным образом симметрично по индексам $j$ и $k$.

Далее, выражение

$$
\begin{gathered}
\sum_{m, n=1}^{L} \mu^{m n} \frac{\partial\left(w_{m}\right)_{k}^{i}}{\partial u^{j}} f_{n}(u)+\Gamma_{r j}^{i}(u) \sum_{m, n=1}^{L} \mu^{m n}\left(w_{m}\right)_{k}^{r}(u) f_{n}(u)= \\
=\sum_{m, n=1}^{L} \mu^{m n}\left(\frac{\partial\left(w_{m}\right)_{k}^{i}}{\partial u^{j}}+\Gamma_{r j}^{i}(u)\left(w_{m}\right)_{k}^{r}(u)\right) f_{n}(u)
\end{gathered}
$$

симметрично по индексам $j$ и $k$, так как соотношение $(14)$, где $b_{k}^{i j}(u)=-g^{i s}(u) \Gamma_{s k}^{j}(u)$, эквивалентно соотношению

$$
\frac{\partial\left(w_{m}\right)_{k}^{i}}{\partial u^{j}}+\Gamma_{r j}^{i}(u)\left(w_{m}\right)_{k}^{r}(u)=\frac{\partial\left(w_{m}\right)_{j}^{i}}{\partial u^{k}}+\Gamma_{r k}^{i}(u)\left(w_{m}\right)_{j}^{r}(u) .
$$

И, наконец, используя соотношение (17), для оставшихся слагаемых выражения (25) получаем

$$
\begin{aligned}
& -\frac{\partial g^{i s}}{\partial u^{j}} \Gamma_{s k}^{p}(u) \frac{\partial h}{\partial u^{p}}-g^{i s}(u) \frac{\partial \Gamma_{s k}^{p}}{\partial u^{j}} \frac{\partial h}{\partial u^{p}}+ \\
& \quad+\sum_{m, n=1}^{L} \mu^{m n}\left(w_{m}\right)_{k}^{i}(u)\left(w_{n}\right)_{j}^{p}(u) \frac{\partial h}{\partial u^{p}}-\Gamma_{r j}^{i}(u) g^{r s}(u) \Gamma_{s k}^{p}(u) \frac{\partial h}{\partial u^{p}}= \\
& =\left(-\frac{\partial\left(g^{i s} \Gamma_{s k}^{p}\right)}{\partial u^{j}}+\sum_{m, n=1}^{L} \mu^{m n}\left(w_{m}\right)_{k}^{i}(u)\left(w_{n}\right)_{j}^{p}(u)-\Gamma_{r j}^{i}(u) g^{r s}(u) \Gamma_{s k}^{p}(u)\right) \frac{\partial h}{\partial u^{p}}
\end{aligned}
$$


а это выражение симметрично по индексам $j$ и $k$, так как соотношение (15), где $b_{k}^{i j}(u)=-g^{i s}(u) \Gamma_{s k}^{j}(u)$, эквивалентно соотношению

$$
\begin{gathered}
-\frac{\partial\left(g^{i s} \Gamma_{s k}^{p}\right)}{\partial u^{j}}+\sum_{m, n=1}^{L} \mu^{m n}\left(w_{m}\right)_{k}^{i}(u)\left(w_{n}\right)_{j}^{p}(u)-\Gamma_{r j}^{i}(u) g^{r s}(u) \Gamma_{s k}^{p}(u)= \\
=-\frac{\partial\left(g^{i s} \Gamma_{s j}^{p}\right)}{\partial u^{k}}+\sum_{m, n=1}^{L} \mu^{m n}\left(w_{m}\right)_{j}^{i}(u)\left(w_{n}\right)_{k}^{p}(u)-\Gamma_{r k}^{i}(u) g^{r s}(u) \Gamma_{s j}^{p}(u) .
\end{gathered}
$$

Для локально-гамильтоновых аффиноров эти важные соотношения (21) и (22) принимают совсем простой вид в плоских локальных координатах метрики $g_{i j}(u)$. Царев [5] доказал, что в этом плоском случае соотношения (21) и (22) являются не только необходимыми, но и достаточными для того, чтобы аффинор был локально-гамильтоновым (т. е. аффинор $V_{j}^{i}(u)$ является локально-гамильтоновым тогда и только тогда, когда существует плоская метрика $g_{i j}(u)$ такая, что выполняются соотношения (21) и (22)). Этот результат был обобщен на случай аффиноров Мохова-Ферапонтова в работе [3]: аффинор $V_{j}^{i}(u)$ является гамильтоновым относительно нелокальной скобки Мохова-Ферапонтова тогда и только тогда, когда существует метрика $g_{i j}(u)$ постоянной кривизны такая, что выполняются соотношения (21) и (22).

Пусть аффинор $V_{j}^{i}(u)$ системы гидродинамического типа (1) удовлетворяет соотношениям (21) и $(22)$, т. е. существует метрика $g_{i j}(u)$ такая, что выполняются соотношения (21) и (22). Если эта система гидродинамического типа диагонализуема, т. е. существуют локальные координаты такие, что аффинор $V_{j}^{i}(u)$ является диагональной матрицей $V_{j}^{i}(u)=V^{i}(u) \delta_{j}^{i}$ в этих специальных локальных координатах (такие локальные координаты называются инвариантами Римана или римановыми инвариантами системы гидродинамического типа, а также соответствующего аффинора), и при этом система является строго гиперболической, т. е. все собственные значения $V^{i}(u), 1 \leqslant i \leqslant N$, аффинора $V_{j}^{i}(u)$ различны $\left(V^{i}(u) \neq V^{j}(u)\right.$ при $\left.i \neq j\right)$, то она может быть проинтегрирована обобщенным методом годографа (см. [5]). В этом случае соотношение (21) эквивалентно условию, что метрика $g_{i j}(u)$ также является диагональной в этих специальных локальных координатах, $g_{i j}(u)=g_{i}(u) \delta_{i j}$, т. е. римановы инварианты являются ортогональными криволинейными координатами в соответствующем псевдоримановом пространстве, а соотношение (22) эквивалентно условию

$$
\frac{\partial V^{i}}{\partial u^{j}}=\frac{\partial \ln \sqrt{g_{i}(u)}}{\partial u^{j}}\left(V^{j}(u)-V^{i}(u)\right), \quad i \neq j .
$$

Следовательно, для собственных значений $V^{i}(u)$ выполнено соотношение

$$
\frac{\partial}{\partial u^{k}}\left(\frac{1}{V^{j}(u)-V^{i}(u)} \frac{\partial V^{i}}{\partial u^{j}}\right)=\frac{\partial}{\partial u^{j}}\left(\frac{1}{V^{k}(u)-V^{i}(u)} \frac{\partial V^{i}}{\partial u^{k}}\right) .
$$

Строго гиперболическая диагональная система гидродинамического типа называется полугамильтоновой, если выполняются соотношения (32) (см. [5]). Царев [5] доказал, что любая строго гиперболическая диагональная полугамильтонова система гидродинамического типа интегрируется обобщенным методом годографа. 


\section{4. ДИАГОНАЛИЗУЕМЫЕ АФФИНОРЫ}

Напомним, что очень важная проблема диагонализуемости для аффинора, которая была поставлена фактически еще Риманом, была полностью решена Хантьесом в работе [6] на основе предшествующих результатов Нийенхейса [7]. Аффинор $V_{j}^{i}(u)$ диагонализуется локальной заменой координат в некоторой области тогда и только тогда, когда он диагонализуется в каждой точке этой области и его тензор Хантьеса тождественно равен нулю. Тензор Хантьеса аффинора $V(u)=\left(V_{j}^{i}(u)\right)$ - это следующий смешанный тензор типа $(1,2)$ (кососимметрическая векторнозначная 2-форма), порождаемый аффинором $V_{j}^{i}(u)$ :

$$
H(X, Y)=N(V(X), V(Y))+V^{2}(N(X, Y))-V(N(X, V(Y)))-V(N(V(X), Y)),
$$

где $X(u)$ и $Y(u)$ - произвольные векторные поля, $V(X)$ - векторное поле $V_{j}^{i}(u) X^{j}(u)$, $N(X, Y)$ - тензор Нийенхейса аффинора $V_{j}^{i}(u)$, т. е. следующий смешанный тензор типа $(1,2)$ (кососимметрическая векторнозначная 2-форма), порождаемый аффинором $V_{j}^{i}(u)$ :

$$
N(X, Y)=[V(X), V(Y)]+V^{2}([X, Y])-V([X, V(Y)])-V([V(X), Y]),
$$

где $[X, Y]$ - коммутатор векторных полей $X(u)$ и $Y(u)$ :

$$
[X, Y]^{i}(u)=X^{k}(u) \frac{\partial Y^{i}}{\partial u^{k}}-Y^{k}(u) \frac{\partial X^{i}}{\partial u^{k}} .
$$

В компонентах тензор Нийенхейса аффинора $V_{j}^{i}(u)$ имеет вид

$$
N_{i j}^{k}(u)=V_{i}^{s}(u) \frac{\partial V_{j}^{k}}{\partial u^{s}}-V_{j}^{s}(u) \frac{\partial V_{i}^{k}}{\partial u^{s}}+V_{s}^{k}(u) \frac{\partial V_{i}^{s}}{\partial u^{j}}-V_{s}^{k}(u) \frac{\partial V_{j}^{s}}{\partial u^{i}},
$$

а тензор Хантьеса аффинора $V_{j}^{i}(u)$ имеет вид

$$
\begin{aligned}
H_{j k}^{i}(u)= & V_{s}^{i}(u) V_{r}^{s}(u) N_{j k}^{r}(u)-V_{s}^{i}(u) N_{r k}^{s}(u) V_{j}^{r}(u)- \\
& -V_{s}^{i}(u) N_{j r}^{s}(u) V_{k}^{r}(u)+N_{s r}^{i}(u) V_{j}^{s}(u) V_{k}^{r}(u) .
\end{aligned}
$$

Напомним также, что инвариантные тензорные условия того, что строго гиперболическая система гидродинамического типа является полугамильтоновой, были найдены в работе [8].

\section{5. НЕЛОКАЛЬНО-БИГАМИЛЬТОНОВЫ СИСТЕМЫ ГИДРОДИНАМИЧЕСКОГО ТИПА}

Мы будем рассматривать бигамильтоновы системы гидродинамического типа. Напомним, что две скобки Пуассона называются согласованными, если любая линейная комбинация этих скобок Пуассона также является скобкой Пуассона [9], а система уравнений, которая является гамильтоновой относительно двух линейно независимых согласованных скобок Пуассона, называется бигамильтоновой. В данной статье мы будем рассматривать системы гидродинамического типа, которые являются бигамильтоновыми относительно двух линейно независимых согласованных невырожденных нелокальных скобок Пуассона гидродинамического типа (6)-(8),

$$
u_{t}^{i}=V_{j}^{i}(u) u_{x}^{j}=\left\{u^{i}(x), H_{1}\right\}_{1}=\left\{u^{i}(x), H_{2}\right\}_{2}, \quad 1 \leqslant i, j \leqslant N,
$$




$$
\begin{gathered}
H_{1}=\int h_{1}(u(x)) d x, \quad H_{2}=\int h_{2}(u(x)) d x \\
\{I, J\}_{1}=\int \frac{\delta I}{\delta u^{i}(x)} P_{1}^{i j} \frac{\delta J}{\delta u^{j}(x)} d x, \quad\{I, J\}_{2}=\int \frac{\delta I}{\delta u^{i}(x)} P_{2}^{i j} \frac{\delta J}{\delta u^{j}(x)} d x \\
P_{1}^{i j}=g_{1}^{i j}(u(x)) \frac{d}{d x}+b_{1, k}^{i j}(u(x)) u_{x}^{k}+ \\
+\sum_{m, n=1}^{L} \mu_{1}^{m n}\left(w_{1, m}\right)_{k}^{i}(u(x)) u_{x}^{k}\left(\frac{d}{d x}\right)^{-1} \circ\left(w_{1, n}\right)_{s}^{j}(u(x)) u_{x}^{s}, \\
P_{2}^{i j}=g_{2}^{i j}(u(x)) \frac{d}{d x}+b_{2, k}^{i j}(u(x)) u_{x}^{k}+ \\
+\sum_{m, n=1}^{L} \mu_{2}^{m n}\left(w_{2, m}\right)_{k}^{i}(u(x)) u_{x}^{k}\left(\frac{d}{d x}\right)^{-1} \circ\left(w_{2, n}\right)_{s}^{j}(u(x)) u_{x}^{s} .
\end{gathered}
$$

Мы будем называть аффинор $V_{j}^{i}(u)$ бигамильтоновым (или нелокально-бигамилътоновым), если этот аффинор является гамильтоновым относительно двух линейно независимых согласованных невырожденных нелокальных скобок Пуассона гидродинамического типа (6)-(8). Это определение является инвариантным.

Прежде всего, отметим, что класс нелокально-бигамильтоновых систем гидродинамического типа (37)-(41) очень богат и имеется много хорошо известных важных примеров таких систем, возникающих в различных приложениях. Явная общая конструкция локально- и нелокально-бигамильтоновых систем гидродинамического типа и соответствующих интегрируемых бигамильтоновых иерархий, порождаемых парами согласованных скобок Пуассона гидродинамического типа, и интегрируемое описание локальных и нелокальных согласованных скобок Пуассона гидродинамического типа были найдены и изучены автором в работах [10]-[22], [4] (см. также [23], [24]).

Здесь мы доказываем, что $(1+1)$-мерные неособые (полупростые) нелокально-бигамильтоновы системы гидродинамического типа являются диагонализуемыми. Напомним, что пара псевдоримановых метрик $g_{1}^{i j}(u)$ и $g_{2}^{i j}(u)$ называется неособой (или полупростой), если собственные значения этой пары метрик, т. е. корни уравнения

$$
\operatorname{det}\left(g_{1}^{i j}(u)-\lambda g_{2}^{i j}(u)\right)=0
$$

различны. В этом случае нелокальная бигамильтонова структура (39)-(41), а также нелокально-бигамильтонова система гидродинамического типа (37)-(41) и соответствующий бигамильтонов аффинор также называются неособыми (или полупросmъıми).

Важно отметить, что, вообще говоря, интегрируемые бигамильтоновы системы гидродинамического типа вовсе не обязательно являются диагонализуемыми, если рассматривать другие классы согласованных скобок Пуассона (даже если обе согласованные скобки Пуассона локальны). Это нетривиальный факт, и мы приведем здесь в подробностях один очень важный пример (см. также [25]-[27]). 


\section{6. НЕДИАГОНАЛИЗУЕМАЯ ИНТЕГРИРУЕМАЯ БИГАМИЛЬТОНОВА СИСТЕМА ГИДРОДИНАМИЧЕСКОГО ТИПА}

Рассмотрим уравнения ассоциативности двумерных топологических квантовых теорий поля (уравнения Виттена-Дийкграафа-Верлинде-Верлинде (ВДВВ), см. [28]-[31]) на функцию (потенциал) $\Phi=\Phi\left(u^{1}, \ldots, u^{N}\right)$ :

$$
\sum_{k=1}^{N} \sum_{l=1}^{N} \frac{\partial^{3} \Phi}{\partial u^{i} \partial u^{j} \partial u^{k}} \eta^{k l} \frac{\partial^{3} \Phi}{\partial u^{l} \partial u^{m} \partial u^{n}}=\sum_{k=1}^{N} \sum_{l=1}^{N} \frac{\partial^{3} \Phi}{\partial u^{i} \partial u^{m} \partial u^{k}} \eta^{k l} \frac{\partial^{3} \Phi}{\partial u^{l} \partial u^{j} \partial u^{n}}
$$

где $\eta^{i j}$ - произвольная постоянная невырожденная симметричная матрица, $\eta^{i j}=$ $\eta^{j i}, \eta^{i j}=$ const, $\operatorname{det}\left(\eta^{i j}\right) \neq 0$. Напомним (см. [28]), что уравнения ассоциативности (43) являются совместными и интегрируемыми методом обратной задачи рассеяния, они обладают богатым множеством нетривиальных решений и каждое их решение $\Phi\left(u^{1}, \ldots, u^{N}\right)$ задает $N$-параметрические деформации специальных фробениусовых алгебр (некоторых специальных коммутативных ассоциативных алгебр с невырожденными инвариантными симметрическими билинейными формами). Действительно, рассмотрим алгебры $A(u)$ в $N$-мерном линейном пространстве с базисом $e_{1}, \ldots, e_{N}$ и умножением (см. [28])

$$
e_{i} \circ e_{j}=c_{i j}^{k}(u) e_{k}, \quad c_{i j}^{k}(u)=\eta^{k s} \frac{\partial^{3} \Phi}{\partial u^{s} \partial u^{i} \partial u^{j}} .
$$

Для всех значений параметров $u=\left(u^{1}, \ldots, u^{N}\right)$ алгебры $A(u)$ коммутативны, $e_{i} \circ e_{j}=$ $e_{j} \circ e_{i}$, а условие ассоциативности

$$
\left(e_{i} \circ e_{j}\right) \circ e_{k}=e_{i} \circ\left(e_{j} \circ e_{k}\right)
$$

в алгебрах $A(u)$ эквивалентно уравнениям ВДВВ (43). Матрица $\eta_{i j}$, обратная матрице $\eta^{i j}, \eta^{i s} \eta_{s j}=\delta_{j}^{i}$, определяет невырожденную инвариантную симметрическую билинейную форму на алгебрах $A(u)$ :

$$
\left\langle e_{i}, e_{j}\right\rangle=\eta_{i j}, \quad\left\langle e_{i} \circ e_{j}, e_{k}\right\rangle=\left\langle e_{i}, e_{j} \circ e_{k}\right\rangle
$$

Напомним (см. [28]), что локально на касательном пространстве в каждой точке любого фробениусова многообразия имеется структура фробениусовой алгебры (44)-(46), которая определяется решением уравнений ассоциативности (43) и гладко зависит от точки. Пусть $N=3$, метрика $\eta_{i j}$ антидиагональна:

$$
\left(\eta_{i j}\right)=\left(\begin{array}{lll}
0 & 0 & 1 \\
0 & 1 & 0 \\
1 & 0 & 0
\end{array}\right)
$$

а $e_{1}$ - единица во фробениусовой алгебре (44)-(46). В этом случае функция (потенциал) $\Phi(u)$ имеет вид

$$
\Phi(u)=\frac{1}{2}\left(u^{1}\right)^{2} u^{3}+\frac{1}{2} u^{1}\left(u^{2}\right)^{2}+f\left(u^{2}, u^{3}\right)
$$


и уравнения ассоциативности (43) для функции $\Phi(u)$ эквивалентны следующему замечательному интегрируемому уравнению Дубровина для функции $f\left(u^{2}, u^{3}\right)$ :

$$
\frac{\partial^{3} f}{\partial\left(u^{3}\right)^{3}}=\left(\frac{\partial^{3} f}{\partial\left(u^{2}\right)^{2} \partial u^{3}}\right)^{2}-\frac{\partial^{3} f}{\partial\left(u^{2}\right)^{3}} \frac{\partial^{3} f}{\partial u^{2} \partial\left(u^{3}\right)^{2}} .
$$

Введем здесь новые независимые переменные: $x=u^{2}, t=u^{3}$. Уравнение (48) принимает вид

$$
f_{t t t}=\left(f_{x x t}\right)^{2}-f_{x x x} f_{x t t} .
$$

Это уравнение связано с квантовыми когомологиями проективной плоскости и классическими проблемами исчислительной геометрии (см. [32]).

В работе [33] (см. также [34], [25]-[27]) автором было доказано, что уравнение (49) эквивалентно интегрируемой недиагонализуемой системе гидродинамического типа

$$
\begin{aligned}
& \left(\begin{array}{l}
a^{1} \\
a^{2} \\
a^{3}
\end{array}\right)_{t}=\left(\begin{array}{ccc}
0 & 1 & 0 \\
0 & 0 & 1 \\
-a^{3} & 2 a^{2} & -a^{1}
\end{array}\right)\left(\begin{array}{l}
a^{1} \\
a^{2} \\
a^{3}
\end{array}\right)_{x}, \\
& a^{1}=f_{x x x}, \quad a^{2}=f_{x x t}, \quad a^{3}=f_{x t t} .
\end{aligned}
$$

Первая гамильтонова структура системы (50), задаваемая скобкой ДубровинаНовикова, была найдена в работе [35]:

$$
\{I, J\}_{1}=\int \frac{\delta I}{\delta a^{i}(x)} M_{1}^{i j} \frac{\delta J}{\delta a^{j}(x)} d x,
$$

$$
\begin{aligned}
M_{1} & =\left(M_{1}^{i j}\right)= \\
& =\left(\begin{array}{ccc}
-3 / 2 & a^{1} / 2 & a^{2} \\
a^{1} / 2 & a^{2} & 3 a^{3} / 2 \\
a^{2} & 3 a^{3} / 2 & 2\left(\left(a^{2}\right)^{2}-a^{1} a^{3}\right)
\end{array}\right) \frac{d}{d x}+\left(\begin{array}{ccc}
0 & a_{x}^{1} / 2 & a_{x}^{2} \\
0 & a_{x}^{2} / 2 & a_{x}^{3} \\
0 & a_{x}^{3} / 2 & \left(\left(a^{2}\right)^{2}-a^{1} a^{3}\right)_{x}
\end{array}\right) .
\end{aligned}
$$

Метрика

$$
\left(g_{1}^{i j}(a)\right)=\left(\begin{array}{ccc}
-3 / 2 & a^{1} / 2 & a^{2} \\
a^{1} / 2 & a^{2} & 3 a^{3} / 2 \\
a^{2} & 3 a^{3} / 2 & 2\left(\left(a^{2}\right)^{2}-a^{1} a^{3}\right)
\end{array}\right)
$$

является плоской, а скобка Пуассона гидродинамического типа (52), (53) является локальной (скобка Дубровина-Новикова). Функционал

$$
H_{1}=\int a^{3} d x
$$

- гамильтониан системы (50):

$$
a_{t}^{i}=\left\{a^{i}(x), H_{1}\right\}_{1} .
$$

Бигамильтонова структура системы (50) была найдена в работе [25] (см. также [26], [27]). Вторая гамильтонова структура системы (50) задается однородной скобкой Дубровина-Новикова третьего порядка:

$$
\{I, J\}_{2}=\int \frac{\delta I}{\delta a^{i}(x)} M_{2}^{i j} \frac{\delta J}{\delta a^{j}(x)} d x,
$$




$$
\begin{aligned}
M_{2}=\left(M_{2}^{i j}\right)= & \left(\begin{array}{ccc}
0 & 0 & 1 \\
0 & 1 & -a^{1} \\
1 & -a^{1} & \left(a^{1}\right)^{2}+2 a^{2}
\end{array}\right)\left(\frac{d}{d x}\right)^{3}+\left(\begin{array}{ccc}
0 & 0 & 0 \\
0 & 0 & -2 a_{x}^{1} \\
0 & -a_{x}^{1} & 3\left(a_{x}^{2}+a^{1} a_{x}^{1}\right)
\end{array}\right)\left(\frac{d}{d x}\right)^{2}+ \\
& +\left(\begin{array}{ccc}
0 & 0 & 0 \\
0 & 0 & 0 \\
0 & 0 & a_{x x}^{2}+\left(a_{x}^{1}\right)^{2}+a^{1} a_{x x}^{1}
\end{array}\right) \frac{d}{d x}
\end{aligned}
$$

Вторая скобка Пуассона (57), (58) является согласованной с первой скобкой Пуассона (52), (53).

Удивительно, но метрика второй гамильтоновой структуры (третьего порядка!)

$$
\left(g_{2}^{i j}(a)\right)=\left(\begin{array}{ccc}
0 & 0 & 1 \\
0 & 1 & -a^{1} \\
1 & -a^{1} & \left(a^{1}\right)^{2}+2 a^{2}
\end{array}\right)
$$

также является плоской. Нелокальный функционал

$$
H_{2}=-\int\left(\frac{1}{2} a^{1}\left(\left(\frac{d}{d x}\right)^{-1} a^{2}\right)^{2}+\left(\left(\frac{d}{d x}\right)^{-1} a^{2}\right)\left(\left(\frac{d}{d x}\right)^{-1} a^{3}\right)\right) d x
$$

является гамильтонианом системы (50):

$$
a_{t}^{i}=\left\{a^{i}(x), H_{2}\right\}_{2} .
$$

\section{7. СОГЛАСОВАННЫЕ МЕТРИКИ \\ И НЕЛОКАЛЬНО-БИГАМИЛЬТОНОВЫ СИСТЕМЫ ГИДРОДИНАМИЧЕСКОГО ТИПА}

Напомним некоторые необходимые основные факты общей теории согласованных метрик [16]-[21]. Две римановы или псевдоримановы контравариантные метрики $g_{1}^{i j}(u)$ и $g_{2}^{i j}(u)$ называются согласованными, если для любой линейной комбинации этих метрик

$$
g^{i j}(u)=\lambda_{1} g_{1}^{i j}(u)+\lambda_{2} g_{2}^{i j}(u),
$$

где $\lambda_{1}$ и $\lambda_{2}-$ произвольные константы такие, что $\operatorname{det}\left(g^{i j}(u)\right) \neq 0$, коэффициенты соответствующих связностей Леви-Чивиты и компоненты соответствующих тензоров римановой кривизны связаны тем же самым линейным соотношением [16]-[18]:

$$
\begin{aligned}
& \Gamma_{k}^{i j}(u)=\lambda_{1} \Gamma_{1, k}^{i j}(u)+\lambda_{2} \Gamma_{2, k}^{i j}(u), \\
& R_{k l}^{i j}(u)=\lambda_{1} R_{1, k l}^{i j}(u)+\lambda_{2} R_{2, k l}^{i j}(u) .
\end{aligned}
$$

Индексы коэффициентов связностей Леви-Чивиты $\Gamma_{j k}^{i}(u)$ и индексы тензоров римановой кривизны $R_{j k l}^{i}(u)$ поднимаются и опускаются с помощью соответствующих им метрик:

$$
\begin{aligned}
\Gamma_{k}^{i j}(u) & =g^{i s}(u) \Gamma_{s k}^{j}(u), \quad \Gamma_{j k}^{i}(u)=\frac{1}{2} g^{i s}(u)\left(\frac{\partial g_{s k}}{\partial u^{j}}+\frac{\partial g_{j s}}{\partial u^{k}}-\frac{\partial g_{j k}}{\partial u^{s}}\right), \\
R_{k l}^{i j}(u) & =g^{i s}(u) R_{s k l}^{j}(u), \\
R_{j k l}^{i}(u) & =\frac{\partial \Gamma_{j l}^{i}}{\partial u^{k}}-\frac{\partial \Gamma_{j k}^{i}}{\partial u^{l}}+\Gamma_{p k}^{i}(u) \Gamma_{j l}^{p}(u)-\Gamma_{p l}^{i}(u) \Gamma_{j k}^{p}(u) .
\end{aligned}
$$


Две римановы или псевдоримановы контравариантные метрики $g_{1}^{i j}(u)$ и $g_{2}^{i j}(u)$ называются почти согласованными, если для любой линейной комбинации этих метрик (62) выполняется соотношение (63) [16]-[18].

Введем аффинор

$$
v_{j}^{i}(u)=g_{1}^{i s}(u) g_{2, s j}(u)
$$

и рассмотрим тензор Нийенхейса этого аффинора

$$
N_{i j}^{k}(u)=v_{i}^{s}(u) \frac{\partial v_{j}^{k}}{\partial u^{s}}-v_{j}^{s}(u) \frac{\partial v_{i}^{k}}{\partial u^{s}}+v_{s}^{k}(u) \frac{\partial v_{i}^{s}}{\partial u^{j}}-v_{s}^{k}(u) \frac{\partial v_{j}^{s}}{\partial u^{i}} .
$$

Теорема 1 [16]-[18]. Две произвольнъе метрики $g_{1}^{i j}(u)$ u $g_{2}^{i j}(u)$ являются почти согласованными тогда и только тогда, когда соответствующий тензор Нийенхейса $N_{i j}^{k}(u)(66)$ тождественно равен нулю.

Предположим, что пара метрик $g_{1}^{i j}(u)$ и $g_{2}^{i j}(u)$ является неособой, т. е. собственные значения этой пары метрик различны. Кроме того, предположим, что метрики $g_{1}^{i j}(u)$ и $g_{2}^{i j}(u)$ являются почти согласованными, т. е. соответствующий тензор Нийенхейса $N_{i j}^{k}(u)(66)$ тождественно равен нулю. В наших статьях [16]-[18] доказано, что в этом случае метрики $g_{1}^{i j}(u)$ и $g_{2}^{i j}(u)$ являются согласованными, т. е. выполняется соотношение (64).

Очевидно, что собственные значения пары метрик $g_{1}^{i j}(u)$ и $g_{2}^{i j}(u)$ совпадают с собственными значениями аффинора $v_{j}^{i}(u)(65)$, а хорошо известно, что если все собственные значения аффинора различны, то из равенства нулю тензора Нийенхейса этого аффинора всегда следует, что существуют специальные локальные координаты (римановы инварианты аффинора $v_{j}^{i}(u)$; мы будем также называть их римановыми инвариантами пары метрик $\left.g_{1}^{i j}(u) u g_{2}^{i j}(u)\right)$ такие, что в этих координатах аффинор приводится к диагональному виду в соответствующей области [7] (см. также [6]).

Следовательно, мы можем считать, что аффинор $v_{j}^{i}(u)$ является диагональным в локальных координатах (римановых инвариантах) $u^{1}, \ldots, u^{N}$, т. е.

$$
v_{j}^{i}(u)=f^{i}(u) \delta_{j}^{i},
$$

где суммирование по повторяющемуся индексу $i$ не производится. В соответствии с нашим предположением собственные значения $f^{i}(u), i=1, \ldots, N$, совпадающие с собственными значениями пары метрик $g_{1}^{i j}(u)$ и $g_{2}^{i j}(u)$, различны:

$$
f^{i}(u) \neq f^{j}(u), \quad i \neq j .
$$

ЛЕмма 1. Если аффинор $v_{j}^{i}(u)$ (65) является диагоналъным в некоторых локальных координатах (римановых инвариантах) и все его собственные значения различны, то в этих локальных координатах метрики $g_{1}^{i j}(u)$ и $g_{2}^{i j}(u)$ такэе обязательно являются диагональными, т.е. в этом случае обе метрики $g_{1}^{i j}(u)$ и $g_{2}^{i j}(u)$ являются диагональными в римановых инвариантах афбинора $v_{j}^{i}(u)$.

Действительно, мы имеем

$$
g_{1}^{i j}(u)=f^{i}(u) g_{2}^{i j}(u)
$$


Из симметрии метрик $g_{1}^{i j}(u)$ и $g_{2}^{i j}(u)$ следует, что для любых индексов $i$ и $j$

$$
\left(f^{i}(u)-f^{j}(u)\right) g_{2}^{i j}(u)=0
$$

где суммирование по повторяющимся индексам не производится, т. е.

$$
g_{2}^{i j}(u)=g_{1}^{i j}(u)=0, \quad i \neq j .
$$

ЛЕмма 2. Пусть афбинор $w_{j}^{i}(u)$ является диагональным в некоторых локальных координатах (римановых инвариантах аффинора $\left.w_{j}^{i}(u)\right) u=\left(u^{1}, \ldots, u^{N}\right)$, m.е. $w_{j}^{i}(u)=\mu^{i}(u) \delta_{j}^{i}$. Тогда справедливы следующие утверждения.

1. Если все собственные значения $\mu^{i}(u), i=1, \ldots, N$, диагонального аффбинора различны, т.е. $\mu^{i}(u) \neq \mu^{j}(u)$ при $i \neq j$, то тензор Нийенхейса этого афбинора тождественно равен нулю тогда и толъко тогда, когда $i$-е собственное значение $\mu^{i}(u)$ зависит только от координаты $u^{i}$.

2. Если все собственные значения диагонального афбинора совпадают, то тензор Нийенхейса тождественно равен нулю.

3. В общем случае произвольного диагонального аффинора $w_{j}^{i}(u)=\mu^{i}(u) \delta_{j}^{i}$ тензор Нийенхейса тождественно равен нулю тогда и только тогда, когда

$$
\frac{\partial \mu^{i}}{\partial u^{j}}=0
$$

для всех индексов $i$ и

Из лемм 1 и 2 следует, что для любой неособой пары почти согласованных метрик всегда существуют локальные координаты (римановы инварианты), в которых метрики имеют специальный вид

$$
g_{2}^{i j}(u)=g^{i}(u) \delta^{i j}, \quad g_{1}^{i j}(u)=f^{i}\left(u^{i}\right) g^{i}(u) \delta^{i j} .
$$

Более того, любая пара диагональных метрик вида (70) для произвольных ненулевых функций $f^{i}\left(u^{i}\right), i=1, \ldots, N$ (они могут быть, например, совпадающими ненулевыми константами, т. е. пара метрик может быть “особой"), является почти согласованной, поскольку соответствующий тензор Нийенхейса всегда тождественно равен нулю для любой пары метрик этого вида. В наших статьях [17], [18] было доказано, что произвольная пара диагональных метрик специального вида (70) для произвольных ненулевых функций $f^{i}\left(u^{i}\right), i=1, \ldots, N$ (пара метрик может быть “особой”), всегда является согласованной, т. е. в этом случае всегда выполняется соотношение (64). Таким образом, мы имеем следующие важные утверждения.

ТЕорема 2 [16]-[18]. Если пара метрик $g_{1}^{i j}(u)$ u $g_{2}^{i j}(u)$ является неособой, т.е. различны корни уравнения

$$
\operatorname{det}\left(g_{1}^{i j}(u)-\lambda g_{2}^{i j}(u)\right)=0
$$

то из равенства нулю тензора Нийенхейса аффинора $v_{j}^{i}(u)=g_{1}^{i s}(u) g_{2, s j}(u)$ следует, что метрики $g_{1}^{i j}(u)$ и $g_{2}^{i j}(u)$ являются согласованными. Таким образом, неособая пара метрик является согласованной тогда и только тогда, когда эта пара метрик является почти согласованной. 
Эта теорема дает оправдание названию класса почти согласованных метрик. Отметим, что, как было показано автором в работах [16]-[18], вообще говоря, почти согласованные метрики не обязательно являются согласованными даже в случае плоских метрик или метрик постоянной кривизны, т. е. в случае скобок Дубровина-Новикова или Мохова-Ферапонтова, но если пара почти согласованных метрик не является согласованной, то эта пара метрик должна быть особой и, следовательно, должна иметь совпадающие собственные значения. Явные примеры почти согласованных метрик, которые не являются согласованными, найдены в работах [16]-[18].

Теорема 3 [16]-[18]. Произвольная неособая пара метрик является согласованной тогда и только тогда, когда существуют локальнъе координать (римановы инварианты $) u=\left(u^{1}, \ldots, u^{N}\right)$ такие, что обе метрики являются диагональными в этих координатах и имеют специальный вид (70), где одна из метрик, в данном случае $g_{2}^{i j}(u)$, является произвольной диагональной метрикой, а $f^{i}\left(u^{i}\right)$, $i=1, \ldots, N,-$ произвольные (вообще говоря, комплексные) ненулевые функции одной переменной. Если некоторые из функиий $f^{i}\left(u^{i}\right), i=1, \ldots, N$, являются ненулевыми совпадающими константами, то пара метрик такого специального вида является особой, но, тем не менее, согласованной.

Обратимся теперь к свойствам согласованных нелокальных скобок Пуассона гидродинамического типа (39)-(41).

Теорема 4 [17]. Если нелокалъные скобки Пуассона гидродинамического типа (39)-(41) являются согласованными, то их метрики $g_{1}^{i j}(u)$ и $g_{2}^{i j}(u)$ являются согласованными.

В работе [2] было доказано, что скобка (6)-(8) является скобкой Пуассона, т. е. она кососимметрическая и удовлетворяет тождеству Якоби, тогда и только тогда, когда:

1) $b_{k}^{i j}(u)=-g^{i s}(u) \Gamma_{s k}^{j}(u)$, где $\Gamma_{s k}^{j}(u)$ - риманова связность, порождаемая контравариантной метрикой $g^{i j}(u)$ (связность Леви-Чивиты);

$2)$ псевдориманова метрика $g^{i j}(u)$ и множество аффиноров $\left(w_{n}\right)_{j}^{i}(u)$ удовлетворяют соотношениям

$$
\begin{array}{cc}
g_{i k}(u)\left(w_{n}\right)_{j}^{k}(u)=g_{j k}(u)\left(w_{n}\right)_{i}^{k}(u), & n=1, \ldots, L, \\
\nabla_{k}\left(w_{n}\right)_{j}^{i}(u)=\nabla_{j}\left(w_{n}\right)_{k}^{i}(u), & n=1, \ldots, L, \\
R_{k l}^{i j}(u)=\sum_{m=1}^{L} \sum_{n=1}^{L} \mu^{m n}\left(\left(w_{m}\right)_{l}^{i}(u)\left(w_{n}\right)_{k}^{j}(u)-\left(w_{m}\right)_{l}^{j}(u)\left(w_{n}\right)_{k}^{i}(u)\right),
\end{array}
$$

и, кроме того, семейство аффиноров $w_{n}(u)$ коммутативно: $\left[w_{m}, w_{n}\right]=0$.

Если нелокальные скобки Пуассона гидродинамического типа (39)-(41) являются согласованными, то из условий согласованности и теоремы Ферапонтова следует, что, во-первых, выполняется соотношение (63), т. е. метрики $g_{1}^{i j}(u)$ и $g_{2}^{i j}(u)$ являются почти согласованными, и, во-вторых, тензор кривизны для метрики $g^{i j}(u)=$ $\lambda_{1} g_{1}^{i j}(u)+\lambda_{2} g_{2}^{i j}(u)$ имеет вид

$$
\begin{aligned}
R_{k l}^{i j}(u)= & \sum_{m=1}^{L_{1}} \sum_{n=1}^{L_{1}} \lambda_{1} \mu_{1}^{m n}\left(\left(w_{1, m}\right)_{l}^{i}(u)\left(w_{1, n}\right)_{k}^{j}(u)-\left(w_{1, m}\right)_{l}^{j}(u)\left(w_{1, n}\right)_{k}^{i}(u)\right)+ \\
& +\sum_{m=1}^{L_{2}} \sum_{n=1}^{L_{2}} \lambda_{2} \mu_{2}^{m n}\left(\left(w_{2, m}\right)_{l}^{i}(u)\left(w_{2, n}\right)_{k}^{j}(u)-\left(w_{2, m}\right)_{l}^{j}(u)\left(w_{2, n}\right)_{k}^{i}(u)\right)= \\
= & \lambda_{1} R_{1, k l}^{i j}(u)+\lambda_{2} R_{2, k l}^{i j}(u),
\end{aligned}
$$


т. е. выполняется соотношение (64) и, следовательно, метрики $g_{1}^{i j}(u)$ и $g_{2}^{i j}(u)$ являются согласованными.

Теорема 5 [17]. Пусть две нелокалъные скобки Пуассона гидродинамического типа (39)-(41) отвечают подмногообразиям с голономной сетъю линий кривизны и заданы в локальных координатах линий кривизны. Если в этом случае соответствующая пара метрик является неособой, то эти нелокальные скобки Пуассона гидродинамического типа являются согласованными тогда и только тогда, когда их метрики являются согласованными.

В этом случае обе метрики $g_{1}^{i j}(u)=g_{1}^{i}(u) \delta^{i j}$ и $g_{2}^{i j}(u)=g_{2}^{i}(u) \delta^{i j}$, а также все операторы Вейнгартена (аффиноры) $\left(w_{1, n}\right)_{j}^{i}(u)=\left(w_{1, n}\right)^{i}(u) \delta_{j}^{i}$ и $\left(w_{2, n}\right)_{j}^{i}(u)=\left(w_{2, n}\right)^{i}(u) \delta_{j}^{i}$ являются диагональными в рассматриваемых локальных координатах. Для любого такого “диагонального" случая условие (72) выполняется автоматически, все операторы Вейнгартена коммутируют, условия (73) и (74) имеют следующий вид:

$$
\begin{gathered}
2 g^{i}(u) \frac{\partial\left(w_{n}\right)^{i}}{\partial u^{k}}=\left(\left(w_{n}\right)^{i}-\left(w_{n}\right)^{k}\right) \frac{\partial g^{i}}{\partial u^{k}}, \quad i \neq k, \\
R_{j i}^{i j}(u)=-R_{i j}^{i j}(u)=\sum_{m=1}^{L} \sum_{n=1}^{L} \mu^{m n}\left(w_{m}\right)^{i}(u)\left(w_{n}\right)^{j}(u), \quad i \neq j, \\
R_{k l}^{i j}(u)=0, \quad k \neq i, \quad k \neq j, \\
R_{k l}^{i j}(u)=0, \quad l \neq i, \quad l \neq j .
\end{gathered}
$$

Из условия, что пара метрик является неособой, и из согласованности этих метрик следует, что соответствующий тензор Нийенхейса тождественно равен нулю и существуют функции $f^{i}\left(u^{i}\right), i=1, \ldots, N$, такие, что

$$
g_{1}^{i}(u)=f^{i}\left(u^{i}\right) g_{2}^{i}(u)
$$

Используя соотношения (75)-(78), несложно доказать, что в этом случае из согласованности метрик следует, что произвольная линейная комбинация рассматриваемых нелокальных скобок Пуассона также является скобкой Пуассона. Таким образом, неособые пары таких "диагональных" нелокальных скобок Пуассона гидродинамического типа (т. е. у этих скобок Пуассона обе метрики $g_{1}^{i j}(u)=g_{1}^{i}(u) \delta^{i j}$ и $g_{2}^{i j}(u)=$ $g_{2}^{i}(u) \delta^{i j}$, а также все операторы Вейнгартена (аффиноры) $\left(w_{1, n}\right)_{j}^{i}(u)=\left(w_{1, n}\right)^{i}(u) \delta_{j}^{i}$ и $\left(w_{2, n}\right)_{j}^{i}(u)=\left(w_{2, n}\right)^{i}(u) \delta_{j}^{i}$ диагональные) являются согласованными тогда и только тогда, когда метрики $g_{1}^{i j}(u)=g_{1}^{i}(u) \delta^{i j}$ и $g_{2}^{i j}(u)=g_{2}^{i}(u) \delta^{i j}$ являются согласованными. Отметим, что сами гамильтоновы операторы $P^{i j}(7)$ в этом “диагональном" случае, вообще говоря, диагональными вовсе не являются. На самом деле, как доказано автором, к теореме 5 сводится и случай произвольной неособой пары согласованных нелокальных скобок Пуассона гидродинамического типа, а именно имеет место следующая важная теорема, позволившая доказать интегрируемость методом обратной задачи рассеяния нелинейных уравнений, описывающих все неособые пары согласованных нелокальных скобок Пуассона гидродинамического типа, в частности в работе [22] удалось построить для этих уравнений пары Лакса со спектральным параметром. 
Теорема 6 [22]. Если пара метрик $g_{1}^{i j}(u)$ u $g_{2}^{i j}(u)$ является неособой, то нелокальные скобки Пуассона гидродинамического типа $\{I, J\}_{1} u\{I, J\}_{2}(39)-(41)$ являются согласованными тогда и только тогда, когда, во-первых, метрики являются согласованными и, во-вторых, обе метрики $g_{1}^{i j}(u), g_{2}^{i j}(u)$ и все афбиноры Вейнгартена $\left(w_{1, n}\right)_{j}^{i}(u),\left(w_{2, n}\right)_{j}^{i}(u)$ могут быть одновременно диагонализованы в некоторой области локальных координат.

Здесь достаточно доказать, что если скобки Пуассона являются согласованными, а соответствующая им пара метрик является неособой, то обе метрики $g_{1}^{i j}(u)$, $g_{2}^{i j}(u)$ и все аффиноры Вейнгартена $\left(w_{1, n}\right)_{j}^{i}(u),\left(w_{2, n}\right)_{j}^{i}(u)$ могут быть одновременно диагонализованы в некоторой области локальных координат. Все остальное следует из предшествующих утверждений. Прежде всего, выше было доказано, что в этом случае метрики $g_{1}^{i j}(u)$ и $g_{2}^{i j}(u)$ являются согласованными. Поскольку пара метрик является неособой, существуют локальные координаты такие, что метрики являются диагональными и имеют специальный вид в локальных координатах (70), где $f^{i}\left(u^{i}\right), 1 \leqslant i \leqslant N$, - функции одной переменной. Функции $f^{i}\left(u^{i}\right)$ являются собственными значениями пары метрик $g_{1}^{i j}(u)$ и $g_{2}^{i j}(u)$, следовательно, по предположению теоремы все они должны быть различными и в случае, если они являются константами (они не могут быть совпадающими константами). Из согласованности скобок Пуассона $\{I, J\}_{1}$ и $\{I, J\}_{2}$ (необходимо рассмотреть соотношение (12) для пучка $\left.\{I, J\}_{1}+\lambda\{I, J\}_{2}\right)$ следует, что

$$
\begin{aligned}
& g_{1}^{i s}\left(w_{2, n}\right)_{s}^{j}=g_{1}^{j s}\left(w_{2, n}\right)_{s}^{i}, \\
& g_{2}^{i s}\left(w_{1, n}\right)_{s}^{j}=g_{2}^{j s}\left(w_{1, n}\right)_{s}^{i} .
\end{aligned}
$$

Кроме того, из соотношения (12) для скобок Пуассона $\{I, J\}_{1}$ и $\{I, J\}_{2}$ мы имеем

$$
\begin{aligned}
& g_{1}^{i s}\left(w_{1, n}\right)_{s}^{j}=g_{1}^{j s}\left(w_{1, n}\right)_{s}^{i}, \\
& g_{2}^{i s}\left(w_{2, n}\right)_{s}^{j}=g_{2}^{j s}\left(w_{2, n}\right)_{s}^{i} .
\end{aligned}
$$

Из (79) и (82) в наших специальных локальных координатах мы получаем

$$
\begin{aligned}
g^{i}\left(w_{2, n}\right)_{i}^{j} & =g^{j}\left(w_{2, n}\right)_{j}^{i}, \\
f^{i}\left(u^{i}\right) g^{i}\left(w_{2, n}\right)_{i}^{j} & =f^{j}\left(u^{j}\right) g^{j}\left(w_{2, n}\right)_{j}^{i},
\end{aligned}
$$

где нет суммирования по повторяющимся индексам. Таким образом,

$$
\left(w_{2, n}\right)_{j}^{i}=\frac{g^{i}}{g^{j}}\left(w_{2, n}\right)_{i}^{j}=\frac{f^{i}\left(u^{i}\right) g^{i}}{f^{j}\left(u^{j}\right) g^{j}}\left(w_{2, n}\right)_{i}^{j},
$$

T. e.

$$
\left(1-\frac{f^{i}\left(u^{i}\right)}{f^{j}\left(u^{j}\right)}\right)\left(w_{2, n}\right)_{i}^{j}=0 .
$$

Следовательно, поскольку все функции $f^{i}\left(u^{i}\right)$ различны, мы получаем

$$
\left(w_{2, n}\right)_{i}^{j}=0, \quad i \neq j .
$$

Аналогично из (80) и (81) мы имеем

$$
\left(w_{1, n}\right)_{i}^{j}=0, \quad i \neq j .
$$


Таким образом, обе метрики $g_{1}^{i j}(u), g_{2}^{i j}(u)$ и все аффиноры Вейнгартена $\left(w_{1, n}\right)_{j}^{i}(u)$, $\left(w_{2, n}\right)_{j}^{i}(u)$ являются диагональными в наших специальных локальных координатах.

Теперь мы можем доказать главную теорему данной статьи.

ТЕОРема 7. Для произволъной неособой (полупростой) нелокально-бигамилътоновой системы гидродинамического типа (37)-(41) существуют локальные координаты (римановы инварианты системы) такие, что все связанные с данной системой матричные дифберенциально-геометрические обгекты, а именно матрица (аффинор) $V_{j}^{i}(u)$ этой системы гидродинамического типа, метрики $g_{1}^{i j}(u)$ u $g_{2}^{i j}(u)$, афбинор $v_{j}^{i}(u)=g_{1}^{i s}(u) g_{2, s j}(u)$ и афбиноры $\left(w_{1, n}\right)_{j}^{i}(u) u\left(w_{2, n}\right)_{j}^{i}(u)$ нелокальной бигамильтоновой структуры этой системы, являются диагональными в этих локальных координатах.

Если мы имеем неособую (полупростую) нелокально-бигамильтонову систему гидродинамического типа (37)-(41), то, как было доказано выше, метрики $g_{1}^{i j}(u)$ и $g_{2}^{i j}(u)$ нелокальной бигамильтоновой структуры этой системы являются согласованными и существуют локальные координаты такие, что $g_{2}^{i j}(u)=g^{i}(u) \delta^{i j}$ и $g_{1}^{i j}(u)=f^{i}\left(u^{i}\right) g^{i}(u) \delta^{i j}$, где $f^{i}\left(u^{i}\right), i=1, \ldots, N,-$ различные ненулевые функции одной переменной (вообще говоря, комплексные), $f^{i}\left(u^{i}\right) \neq f^{j}\left(u^{j}\right), i \neq j$. Выше было также доказано, что аффиноры $\left(w_{1, n}\right)_{j}^{i}(u)$ и $\left(w_{2, n}\right)_{j}^{i}(u)$ нелокальной бигамильтоновой структуры этой системы являются диагональными в этих локальных координатах. Докажем теперь, что матрица (аффинор) $V_{j}^{i}(u)$ этой системы также является диагональной в этих специальных локальных координатах, т. е. докажем, что эти специальные локальные координаты являются римановыми инвариантами рассматриваемой системы гидродинамического типа.

Действительно, в этих локальных координатах из соотношений (21) мы имеем

$$
g_{i}(u) V_{j}^{i}(u)=g_{j}(u) V_{i}^{j}(u), \quad f_{i}\left(u^{i}\right) g_{i}(u) V_{j}^{i}(u)=f_{j}\left(u^{j}\right) g_{j}(u) V_{i}^{j}(u),
$$

где нет суммирования по повторяющимся индексам.

Следовательно,

$$
V_{j}^{i}(u)=\frac{g_{j}(u)}{g_{i}(u)} V_{i}^{j}(u)=\frac{f_{j}\left(u^{j}\right) g_{j}(u)}{f_{i}\left(u^{i}\right) g_{i}(u)} V_{i}^{j}(u),
$$

T. e.

$$
\frac{g_{j}(u)}{g_{i}(u)} V_{i}^{j}(u)=\frac{f_{j}\left(u^{j}\right) g_{j}(u)}{f_{i}\left(u^{i}\right) g_{i}(u)} V_{i}^{j}(u) .
$$

Таким образом,

$$
\left(f_{i}\left(u^{i}\right)-f_{j}\left(u^{j}\right)\right) V_{i}^{j}(u)=0
$$

T. e.

$$
V_{j}^{i}(u)=0, \quad i \neq j,
$$

и диагонализуемость произвольной неособой (полупростой) нелокально-бигамильтоновой системы гидродинамического типа доказана:

$$
V_{j}^{i}(u)=V^{i}(u) \delta_{j}^{i}
$$

Таким образом, для произвольной неособой (полупростой) нелокально-бигамильтоновой системы гидродинамического типа построен полный набор римановых инвариантов. 
Удивительно и очень интересно то, что построенные римановы инварианты произвольной неособой (полупростой) нелокально-бигамильтоновой системы гидродинамического типа полностью определяются метриками $g_{1}^{i j}(u)$ и $g_{2}^{i j}(u)$ бигамильтоновой структуры и не зависят ни от аффиноров Вейнгартена $\left(w_{1, n}\right)_{j}^{i}(u)$ и $\left(w_{2, n}\right)_{j}^{i}(u)$ бигамильтоновой структуры, ни от гамильтонианов $H_{1}$ и $H_{2}$ бигамильтоновой системы.

Диагонализуемость неособых (полупростых) локально-бигамильтоновых систем гидродинамического типа (37)-(41) была отмечена в работе [24]; это свойство немедленно следует из теории неособых (полупростых) пар согласованных плоских метрик [16]-[23].

Отметим, что из доказательства не следует, что $V^{i}(u) \neq V^{j}(u)$ при $i \neq j$, т. е. произвольная неособая (полупростая) нелокально-бигамильтонова система гидродинамического типа не обязана быть строго гиперболической, хотя нам неизвестны примеры таких систем, у которых некоторые собственные значения (характеристические скорости) $V^{i}(u)$ совпадают. Мы предполагаем, что такие системы существуют, и было бы очень интересно найти примеры неособых (полупростых) нелокально-бигамильтоновых систем гидродинамического типа, у которых есть совпадающие собственные значения $V^{i}(u)$.

Отметим также, что условие неособости пары метрик очень существенно для нашего утверждения о диагонализуемости системы, и мы предполагаем, что существуют недиагонализуемые особые нелокально-бигамильтоновы системы гидродинамического типа. Было бы также очень интересно найти примеры таких систем.

Благодарности. Работа выполнена при финансовой поддержке Института математики имени Макса Планка (Бонн, Германия), Российского фонда фундаментальных исследований (гранты № 08-01-00464, 09-01-12148-офи-м) и Программы поддержки ведущих научных школ (грант НШ-1824.2008.1).

\section{Список литературы}

[1] Б. А. Дубровин, С. П. Новиков, ДАН СССР, 270:4 (1983), 781-785.

[2] Е. В. Ферапонтов, Функи. анализ и его прил., 25:3 (1991), 37-49.

[3] О. И. Мохов, Е. В. Ферапонтов, УМН, 45:3(273) (1990), 191-192.

[4] О. И. Мохов, Функи. анализ и его прил., 40:1 (2006), 14-29, arXiv: math.DG/0406292.

[5] С. П. Царев, Изв. АН СССР. Сер. матем., 54:5 (1990), 1048-1068.

[6] J. Haantjes, Indag. Math., 17:2 (1955), 158-162.

[7] A. Nijenhuis, Indag. Math., 13:2 (1951), 200-212.

[8] М. В. Павлов, С. И. Свинолупов, Р. А. Шарипов, Функи. анализ и его прил., 30:1 (1996), $18-29$.

[9] F. Magri, J. Math. Phys., 19:5 (1978), 1156-1162.

[10] О. И. Мохов, ТМФ, 132:1 (2002), 60-73, arXiv: math.DG/0201242.

[11] О. И. Мохов, УМН, 57:1(343) (2002), 157-158.

[12] О. И. Мохов, УМН, 57:5(347) (2002), 157-158.

[13] О. И. Мохов, Функи. анализ и его прил., 37:2 (2003), 28-40, arXiv: math.DG/0201223.

[14] O. I. Mokhov, "Frobenius manifolds as a special class of submanifolds in pseudo-Euclidean spaces", Geometry, Topology, and Mathematical Physics, Amer. Math. Soc. Transl. Ser. 2, 224, eds. V. M. Buchstaber, I. M. Krichever, AMS, Providence, RI, 2008, 213-246, arXiv: 0710.5860 .

[15] О. И. Мохов, ТМФ, 133:2 (2002), 279-288, arXiv: math.DG/0201281. 
[16] О. И. Мохов, УМН, 55:4(334) (2000), 217-218.

[17] О. И. Мохов, Функи. анализ и его прил., 35:2 (2001), 24-36, arXiv: math.DG/0005051.

[18] O. I. Mokhov, J. Appl. Math., 2:7 (2002), 337-370, arXiv: math.DG/0201224.

[19] О. И. Мохов, УМН, 56:2(338) (2001), 221-222.

[20] О. И. Мохов, ТМФ, 130:2 (2002), 233-250, arXiv: math.DG/0005081.

[21] О. И. Мохов, Функи. анализ и его прил., 36:3 (2002), 36-47, arXiv: math.DG/0201280.

[22] О. И. Мохов, ТМФ, 138:2 (2004), 283-296, arXiv: math.DG/0202036.

[23] E. V. Ferapontov, J. Phys. A, 34:11 (2001), 2377-2388, arXiv: math.DG/0005221.

[24] B. Dubrovin, Si-Qi Liu, Y. Zhang, Comm. Pure Appl. Math., 59:4 (2006), 559-615, arXiv: math/0410027.

[25] E. V. Ferapontov, C. A.P. Galvão, O. I. Mokhov, Y. Nutku, Comm. Math. Phys., 186:3 (1997), 649-669.

[26] О. И. Мохов, УМН, 53:3(321) (1998), 85-192.

[27] О.И. Мохов, Симплектическая и пуассонова геометрия на пространствах петель гладких многообразий и интегрируемые уравнения, Ин-т компьютерных исследований, Москва, Ижевск, 2004.

[28] B. Dubrovin, "Geometry of 2D topological field theories", Integrable Systems and Quantum Groups, Lecture Notes in Math., 1620, eds. M. Francaviglia, S. Greco, Springer, Berlin, 1996, 120-348, arXiv: hep-th/9407018.

[29] E. Witten, Nucl. Phys. B, 340:2-3 (1990), 281-332.

[30] E. Witten, "Two-dimensional gravity and intersection theory on moduli space", Surveys in Differential Geometry, Proc. of the Conference on Geometry and Topology (April 27-29, 1990), v. 1, eds. Shing-Tung Yau, Chuan-Chih Hsiung, Lehigh Univ., Bethlehem, PA, 1991, $243-310$.

[31] R. Dijkgraaf, H. Verlinde, E. Verlinde, Nucl. Phys. B, 352:1 (1991), 59-86.

[32] M. Kontsevich, Yu. Manin, Comm. Math. Phys., 164:3 (1994), 525-562, arXiv: hep-th/9402147.

[33] O. I. Mokhov, "Symplectic and Poisson geometry on loop spaces of manifolds and nonlinear equations", Topics in Topology and Mathematical Physics, Amer. Math. Soc. Transl. Ser. 2, 170, ed. S. P. Novikov, AMS, Providence, RI, 1995, 121-151, arXiv: hep-th/9503076.

[34] O.I. Mokhov, "Poisson and symplectic geometry on loop spaces of smooth manifolds", Geometry from the Pacific Rim, eds. A. J. Berrick, B. Loo, H.-Y. Wang, de Gruyter, Berlin, 1997, 285-309.

[35] О. И. Мохов, Е. В. Ферапонтов, Функи. анализ и его прил., 30:3 (1996), 62-72, arXiv: hep-th/9505180.

Поступила в редакцию 14.10.2010 